\title{
Responsabilidad social de las empresas agrícolas y agroindustriales aguacateras de Uruapan, Michoacán, y sus implicaciones en la competitividad
}

\author{
Katia Beatriz Villafán \\ Vidales \\ Facultad de Economía "Vasco \\ de Quiroga", Universidad \\ Michoacana de San Nicolás de \\ Hidalgo \\ kbvillafan@fevaq.net
}

Dante Ariel Ayala Ortiz

Facultad de Economía

"Vasco de Quiroga",

Universidad Michoacana de

San Nicolás de Hidalgo

daao@fevaq.net

\section{Resumen}

En Michoacán las actividades agrícola y agroindustrial son muy importantes económica y socialmente; Uruapan destaca por su actividad aguacatera, pues representa la base económica de este municipio. A pesar de los beneficios económicos, la agroindustria del aguacate en esta región ha generado daños, principalmente en el aspecto medioambiental. En esta investigación se plantea la Responsabilidad Social Empresarial (RSE) como una contribución para solucionar esta problemática. El objetivo es conocer el nivel de RSE de las empresas aguacateras de Uruapan; para ello se elaboró un índice de RSE basado en el modelo Cemefi y el método de valoración multicriterio de proceso analítico jerárquico (AHP). Se entrevistó y evaluó a doce empresas entre julio y diciembre de 2010. En los resultados se obtuvo un nivel medio de RSE (51.58\%), un nivel alto en calidad de vida (63.22\%), un nivel medio en ética empresarial $(47.85 \%)$, un nivel bajo en vinculación con la comunidad $(22.95 \%)$ y un nivel bajo en medio ambiente $(24.15 \%)$. De cada variable también se identificaron los aspectos que generan efectos positivos o que inhiben la competitividad. Finalmente, se dan las recomendaciones para elaborar una estrategia de RSE para ser aplicada en el sector aguacatero de la región.

Palabras clave: AHP, competitividad, Michoacán, RSE, sector aguacatero, Uruapan 


\title{
Corporate Social Responsibility in agricultural and agroindustrial avocado companies from Uruapan, Michoacán, and its competitiveness implications
}

\begin{abstract}
In Michoacán farming and agribusiness are great economic and social activities. Uruapan is the main avocado producer municipality in the state and avocado industry is the basis for the economy of this municipality. Despite the economic benefits, avocado industry has also caused damage, mainly in the environmental aspect. This research focuses on Corporate Social Responsibility (CSR) as a way to contribute to solve this problem. The aim of this paper is to determine the CSR level of avocado companies from Uruapan. In order to know this level, a CSR index was built based on the Cemefi model and the analytic hierarchy process (AHP). Twelve avocado companies were interviewed and evaluated during july and december 2010. As results we find a CSR level of $51.58 \%$, which is translated as a medium level. We also find a high quality of life level (63.22\%), a medium business ethics level $(47.85 \%)$, a low involvement with the community level $(22.95 \%)$ and also a low environmental level $(24.15 \%)$. It has also been identified, from each variable, the main issues that generate competitiveness or that inhibit it. Finally, we give some recommendations in order to develop a CSR strategy to be applied in the sector.
\end{abstract}

Keywords: AHP, competitiveness, Michoacán, CSR, avocado sector, Uruapan

\section{Introducción}

En México, y particularmente en el estado de Michoacán, la actividad agrícola y agroindustrial son muy importantes económica y socialmente. La región de Uruapan es una de las principales zonas agrícolas del estado, debido a que su ubicación geográfica y condiciones climáticas hacen de ella el lugar idóneo para el cultivo de durazno, papaya, limón, nopal, mango, frutas exóticas, pero principalmente aguacate.

La actividad aguacatera representa la base de la economía de esta región. Actualmente el aguacate ocupa 16588 hectáreas de cultivo (20\% de la superficie total del municipio), lo que genera 8195 empleos directos, 12106 empleos estacionales y 32608 empleos indirectos permanentes. Además, se ubican 105 de las 152 empresas empacadoras en el estado que envasan fruta para el mercado nacional e internacional. Aunque esta actividad ha generado importantes beneficios económicos para la región, también ha generado externalidades negativas, principalmente en el aspecto medioambiental (CONANP, 2006). 
Si bien existe poca literatura teórica acerca del impacto económico y ecológico que ha generado la expansión de este cultivo en la región (Barsimantov y Navia, 2008), existe evidencia de dos problemáticas específicas que la actividad aguacatera ha generado: por un lado, la pérdida de biodiversidad que conlleva el monocultivo de aguacate; por otro, los efectos en la salud de los jornaleros y las poblaciones vecinas por el uso excesivo de agroquímicos, así como la contaminación de importantes recursos naturales como es el caso de la subcuenca del río Cupatitzio, principal recurso hídrico de la región. En gran medida, las empresas del sector son responsables de este problema; sin embargo, muy poco se he hecho para dar una solución a pesar de que el sostenimiento a mediano y largo plazo de su actividad depende de la conservación de este importante recurso hídrico. El gobierno ha generado las condiciones para diseñar y hacer cumplir las leyes ambientales, pero esto no ha sido suficiente, por lo que se requiere de una solución que involucre a los principales responsables, es decir, a las empresas.

En este sentido, en este trabajo se plantea el impulso de una estrategia de Responsabilidad Social Empresarial (RSE) para las empresas agrícolas y agroindustriales del municipio de Uruapan con la finalidad de que, al incluir la gestión de los aspectos ambientales y sociales en sus actividades diarias, éstas contribuyan al sostenimiento de la actividad con una visión sustentable.

Con la RSE se generan beneficios al interior de la empresa, tales como una mejor calidad del entorno laboral, relaciones más cordiales y sólidas con los grupos de interés, entre otros; asimismo, beneficios al exterior de ella mediante una mayor vinculación con la comunidad y un mayor involucramiento con temas medioambientales. Estos beneficios pueden ser traducidos como ventajas competitivas sobre el resto de los competidores.

Para el desarrollo de una estrategia sectorial de RSE es importante conocer el punto de partida. Así, el objetivo de esta investigación es conocer cuál es el nivel de RSE de las empresas agrícolas y agroindustriales del municipio de Uruapan, Michoacán, y determinar a partir de los resultados obtenidos cuáles son las implicaciones que tiene dicho nivel en su competitividad.

Dado que existen pocos trabajos que se hayan hecho con este perfil, los autores sostienen que en el presente se buscan especificar las propiedades, características y los perfiles de las empresas agroindustriales del aguacate en Uruapan en relación con la RSE. En este sentido, una importante aportación del presente trabajo es el 
desarrollo de un método de medición más preciso de la RSE; a partir del modelo del Centro Mexicano de Filantropía, A.C., al que se incorpora un sistema de ponderación diferenciada con base en la importancia relativa que se logró establecer empleando un ejercicio de análisis multicriterio, conocido como proceso analítico jerárquico.

El documento se presenta en cinco partes. Primero, se presentan los fundamentos teóricos de la RSE y la competitividad, donde se establece los aspectos que relacionan a ambas variables. En seguida, se presenta la metodología utilizada para medir la RSE de las empresas agrícolas y agroindustriales de aguacate del municipio de Uruapan, Michoacán, la cual consiste en un índice de RSE elaborado por los autores a partir del modelo propuesto por el Centro Mexicano para la Filantropía, A.C (Cemefi), incorporando un método de valoración multicriterio del tipo proceso analítico jerárquico (AHP). Después, se presentan los resultados obtenidos en el índice, así como de cada uno de los cuatro planos analizados por el modelo (i.e.calidad de vida, ética empresarial, vinculación con la comunidad y medio ambiente), analizando las implicaciones de estos resultados sobre la competitividad del sector. Posteriormente, se presenta una discusión de los resultados obtenidos. Por último, las conclusiones y recomendaciones de esta investigación.

\section{Responsabilidad Social Empresarial y competitividad: fundamentos teóricos}

La Responsabilidad Social Empresarial (RSE) tiene sus orígenes en 1953 con la publicación Social Responsibility of the Businessman del economista norteamericano Howard R. Bowen. Desde sus inicios hasta el día de hoy han surgido una gran diversidad de enfoques teóricos entre los que se encuentran el moralista, contractual y utilitarista, o el instrumental, político, integrador y ético (Gendron, 2000; Garriga y Melé, 2004).

Conforme han ido apareciendo nuevos enfoques, la diversidad de definiciones sobre la RSE ha aumentado considerablemente debido a su carácter multidisciplinario. Mientras algunos autores establecen que no existe un consenso entre contenido, fundamentos teóricos o implicaciones sociales y organizacionales de dicha responsabilidad (Gendron, 2000), otros han realizado esfuerzos para homogenizar el concepto sin lograrlo del todo completamente (Mardensen, 2006; McWilliams, 2006). 
El reciente interés de organismos internacionales, de carácter público y privado, por este tema ha influido en gran medida a que éstos participen activamente en la homogenización del concepto. En particular, la Organización Internacional para la Estandarización comenzó a trabajar desde el año 2005 en la elaboración de la norma ISO 26000 sobre RSE y ha reunido desde entonces a más de 450 expertos y 210 observadores de 99 países miembros y 42 organizaciones (ISO, 2010). Esta organización define la RSE como:

la responsabilidad de una organización ante los impactos que sus decisiones y actividades ocasionan en la sociedad y el medio ambiente mediante un comportamiento ético y transparente que contribuya al desarrollo sostenible, incluyendo la salud y el bienestar de la sociedad, que toma en consideración las expectativas de sus partes interesadas, que cumpla con la legislación aplicable y sea coherente con la normativa internacional de comportamiento, y que está integrada en toda la organización y se lleva a la práctica en sus relaciones (ISO, 2010).

Dicha definición, que ha comenzado a ser la referencia para las empresas en el nivel mundial, tiene su base en el enfoque estratégico de la RSE, el cual también ha tenido una gran aceptación en el mundo académico y empresarial.

De acuerdo con la clasificación de Gendron (2000), el enfoque estratégico forma parte de la visión utilitarista de la $\mathrm{RSE}^{1}$. Por lo que, en la clasificación de Garriga y Melé (2004) este enfoque emerge de las denominadas teorías instrumentales. ${ }^{2}$ El enfoque utilitarista se define como "argumentos instrumentales a favor de la responsabilidad social que asumen una relación positiva entre el comportamiento socialmente responsable y el nivel de desarrollo económico de la empresa" (Jones, 1996); considera que a mediano y largo plazo lo que es bueno para la sociedad es igualmente bueno para la empresa, lo cual supone que: lo que es bueno para la empresa lo es también para la sociedad. Los argumentos en los que se basa este enfoque son los siguientes: 1) la empresa socialmente responsable puede aprovechar las oportunidades de mercado provocadas por la transformación de valores sociales y medioambientales; 2) un comportamiento socialmente responsable puede generar una ventaja competitiva; 3 ) una estrategia de responsabilidad social permite

${ }^{1}$ Para profundizar en la clasificación véase Gendron Corinne (2000).

${ }^{2}$ Para mayor detalle de la clasificación véase Garriga y Melé (2004). 
a la empresa anticiparse y enfrentar ciertas medidas legislativas (Gendron, 2000). Es en dicha perspectiva que se comprende la versión moderna de la filantropía de la empresa, que se refiere a la transformación de la actividad caritativa hacia una actividad estratégica (Pasquero, 1995) en la medida en que pretende mejorar la imagen pública de la empresa y obtener una ventaja competitiva.

Las teorías instrumentales, por su parte, consideran a la RSE como una herramienta estratégica para lograr los objetivos económicos y, en última instancia, la creación de la riqueza. Para Garriga y Melé (2004), la RSE se entiende como una herramienta o instrumento estratégico de la empresa que ayuda a obtener mayores ganancias, incrementar su competitividad o adelantarse a ciertas medidas legislativas. Estas teorías, que han tenido una gran aceptación en el mundo de los negocios, pueden ser clasificadas de acuerdo con el objetivo económico propuesto de la siguiente manera: 1) las teorías cuyo objetivo es la maximización del valor de los accionistas medido por el precio de las acciones (Friedman, 1970; Jensen, 2000); 2) las investigaciones que intentan encontrar una relación positiva entre RSE y desempeño financiero (Griffin y Mahon, 1997; Margolis y Walsh, 2003; Gómez, 2006); y 3) las teorías cuyo objetivo estratégico es lograr una ventaja competitiva (Porter y Kramer, 2006; Hart, 1995; Lizt, 1996; Prahalad y Hammond, 2002; Hart y Christensen, 2002; Prahalad, 2002; Garriga y Melé, 2004). Este último conjunto de teorías "de objetivo estratégico" ha comenzado a tener un gran número de seguidores y al mismo tiempo ha sido muy criticado por su enfoque estrictamente neoliberal.

De acuerdo con la teoría de la ventaja competitiva, una empresa busca mantener su liderazgo en el mercado con base en tres estrategias genéricas: costos, enfoque y diferenciación (Porter, 2002). Así, la RSE — dentro de la perspectiva competitiva - es considerada una estrategia de diferenciación. Una empresa que establece una estrategia de este tipo busca competir, ya sea en el producto, ya sea en el servicio, para que clientes específicos perciban un mayor valor en él gracias a la mejora de alguna de sus características. Pero la diferenciación es una estrategia que se basa en el efecto causado sobre aquellas variables que los clientes y consumidores consideran importantes a la hora de tomar su decisión de compra. Por lo tanto, cualquier acción de RSE llevada a cabo por una empresa, por mucho beneficio social que genere, no podría considerarse como una fuente de ventaja competitiva si el mercado no la valorase positivamente y estuviera dispuesto a compensar. 
En este mismo sentido, temas como la equidad de marca, la reputación o la innovación, el cuidado al medio ambiente, los cuales están fuertemente influenciados por la RSE, son aspectos que cada vez más son considerados por los consumidores para elegir sus productos $\mathrm{y}$, por lo tanto, comienzan a verse como nuevos factores determinantes de la competitividad empresarial (Villanova, 2009).

La RSE genera importantes beneficios en las condiciones laborales de los trabajadores, la relación de la empresa con los stakeholders, la imagen y reputación corporativa, así como en la transparencia empresarial, con los cuales se puede obtener una ventaja competitiva.

Las condiciones laborales - más dignas y justas - de los trabajadores ayudan a incrementar su nivel de satisfacción laboral, con lo que incrementan su productividad, que según Propenko (1991) es la relación entre la producción obtenida por un sistema de producción y los recursos utilizados para obtenerla. En este proceso intervienen factores humanos materiales y externos (Pedraza, 2004). Cuando el trabajador se encuentra satisfecho con su trabajo se evitan costos derivados por la rotación de personal, con lo que se obtiene un efecto positivo en la productividad de la empresa (Becchetti, 2004).

La RSE también genera una mejora en las relaciones entre los distintos stakeholders o grupos de interés para la empresa. Freeman (1984) establece que los empresarios deben ajustar sus políticas para satisfacer a un mayor número de grupos interesados de la empresa, no solamente los accionistas. Estos stakeholders o grupos de interés incluyen a los trabajadores, clientes, proveedores y organizaciones de la comunidad (Freeman, 1984). El correcto entendimiento y administración de las partes interesadas que sugiere la RSE generará una mejor comprensión del entorno competitivo (Donaldson y Preston, 1995; Freeman, 1984; Kay, 1993). Además de los beneficios mencionados anteriormente, la RSE tiene un efecto positivo en la imagen y reputación de la empresa. Ambos aspectos son considerados un activo intangible (Scheitz y Epstein, 2005) que influye en los consumidores, la demanda laboral y la inversión principalmente.

El patrón de consumo actualmente está relacionado con el desarrollo de negocios sostenibles que incluye las perspectivas ambientales, sociales y legales. Nuevos estudios empíricos muestran que un gran número de consumidores están dispuestos a pagar un sobreprecio por productos que son elaborados por empresas cuyas condiciones laborales son estrictamente ambientales y no discriminatorias (Jus- 
cius, 2008). De esta manera, la empresa que actúa de manera socialmente responsable tendrá un impacto positivo en la imagen que tenga el consumidor sobre la compañía, puesto que ésta cumplirá con sus expectativas.

Actualmente, por el lado de la demanda laboral, los nuevos profesionistas que se incorporan al mercado de trabajo comienzan a evaluar a las empresas en términos de su RSE, específicamente por lo que corresponde a las condiciones laborales. Como ejemplo de ello se encuentra el índice de clima laboral del Great Place to Work Institute, el cual evalúa a las empresas en cinco aspectos: credibilidad, respeto, imparcialidad, orgullo y camaradería (Great Place to Work, 2011).

La reputación y la imagen también son aspectos clave para la administración del riesgo. Cada vez hay un mayor número de accionistas monitoreando y evaluando a las empresas en términos de su RSE. Como ejemplo se encuentran los rankings o índices relacionados con la capacidad de innovación, la equidad de marca (Business Week, 2007), la rendición de cuentas (Fortune, 2007), los derechos humanos (Business and Human Rights Resource Center, 2007), entre otros.

Por último, la RSE permite mejorar la transparencia de las empresas mediante un proceso administrativo de rendición de cuentas (Elkinton, 1998; Valor, 2005). El principio de transparencia establece que una organización debería ser transparente en sus decisiones y actividades que impactan sobre otros. Una empresa transparente supone que debe entregar información de manera clara, equilibrada y veraz, en un grado razonable y suficiente, sobre las políticas, decisiones y actividades por las cuales es responsable; incluyendo sus actuales y potenciales impactos sobre la sociedad y el medio ambiente. Una empresa, entonces, debe ser transparente sobre la manera en que son tomadas, implementadas y revisadas sus decisiones incluyendo al menos lo siguiente: 1) la definición de roles, responsabilidades, rendición de cuentas y autoridades a través de las diferentes funciones de la organización; 2) las normas y criterios contra los cuales la organización evalúa su propio desempeño; 3) el propósito, la naturaleza de sus actividades y lugar donde se realizan; y 4) el impacto de sus decisiones y actividades sobre otros (Villaverde, 2008).

En resumen, la RSE se ha convertido en un factor esencial para la competitividad porque es una estrategia de diferenciación de la empresa que involucra aspectos sociales y ambientales, tanto en ésta como en los diversos grupos de interés. Mediante la RSE se mejoran las condicionales laborales de los trabajadores y las relaciones con los diferentes grupos de interés; con ello se ayuda a reducir costos y 
a mejorar la productividad de la empresa. Se genera, además, un impacto positivo en la imagen y reputación de la empresa entre los distintos grupos de interés y mejora la transparencia y la confianza que supone al rendir cuentas a cada uno de los grupos de interés.

En la figura 1 se resume la relación que los autores identifican entre la RSE y la competitividad a partir de la revisión de las diversas teorías y enfoques aquí presentados; los cuales dan cuenta de la estrecha vinculación que existe entre ambos conceptos.

\section{Figura 1}

\section{Relación teórica entre la RSE y la competitividad}

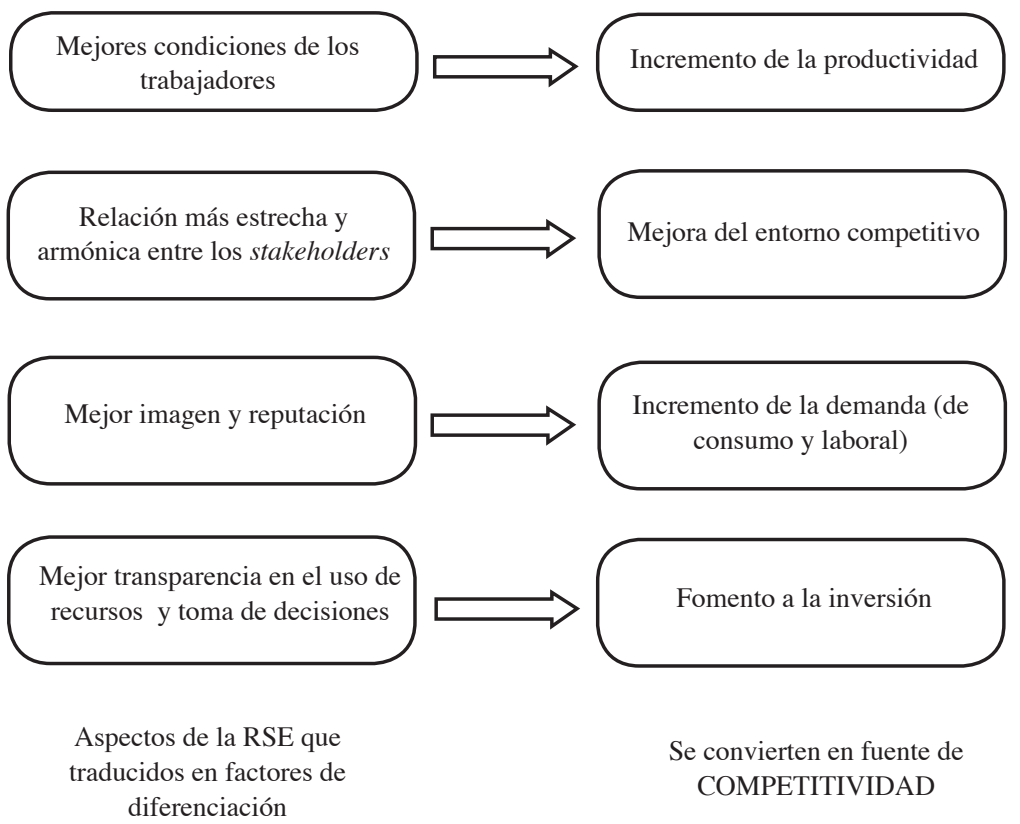

Así, se observa cómo existen una serie de aspectos de la RSE entre los que destacan, por ejemplo, el procurar mejores condiciones a los trabajadores, promover una relación más estrecha y armónica con los stakeholders, mejorar la imagen y reputación de la empresa, y dar mayor transparencia al uso de los recursos y toma 
de decisiones; los que al ser traducidos como factores de diferenciación se convierten en fuente de competitividad para dichas empresas. Lo anterior da lugar al incremento de la productividad del factor trabajo y a la mejora del entorno competitivo, lo que a su vez hace que se incremente la demanda de los productos de la empresa y que un mayor número de trabajadores potenciales demanden trabajar en ella; de igual forma, una mayor transparencia propicia el fomento a la inversión productiva y financiera.

En el caso específico de la agroindustria existen unas investigaciones que describen los procesos de establecimiento de RSE, otras que la cuestionan y algunas más presentadas como casos de estudio de empresas internacionales. Desde hace algunos años se ha observado un amplio bagaje de iniciativas de RSE en forma de certificaciones sociales y ambientales, tanto de producto como de empresa u organización. Estas certificaciones han estado conformadas por indicadores con los que ha sido posible medir el desempeño de RSE de una organización. Como ejemplos tenemos a las certificaciones orgánicas Demeter biodinamica, Nature y Progrès, Northwest California Clean, Core Values Northeast y The Food Alliance, Shade Coffe Campagne, Wholesome Food Association; así como las certificaciones sociales: comercio justo, bio-justo y certificación SA8000. En general, el objetivo de estas certificaciones es promover mejores condiciones de trabajo y se basa en los acuerdos internacionales sobre las condiciones laborales, incluyendo temas tales como justicia social, derechos de los trabajadores, etcétera (Ouellet et al., 2004).

No obstante, en los últimos años ha habido una proliferación de iniciativas que han sido utilizadas para medir la RSE en empresas de todos los sectores, incluyendo la agroindustria, entre las que podernos mencionar el Global Reporting Iniciative (GRI), el Pacto Global de la ONU, el EMAS, la Declaración de la OIT, los Principios de la OECD, AA1000 y el actual ISO 26000.

Al respecto, Kissinger (2012) analiza cómo la RSE en el sector agrícola puede contribuir a lograr la reducción de gases de efecto invernadero. En su investigación no se centra en medir la RSE, más bien toma como referencia el desempeño de RSE con base en la memoria de sostenibilidad de las compañías, la cual se ha sido elaborada con base en el GRI. Al igual que Kissinger, otros investigadores también han utilizado esta herramienta como indicador del desempeño de RSE para llevar a cabo sus investigaciones (Brine, Brown y Hackett, 2007). 
En México es escaso el número de empresas que cuentan con una memoria de sostenibilidad y la referencia para medir el desempeño de RSE ha sido principalmente el distintivo Empresa Socialmente Responsable (ESR) otorgado por el Centro Mexicano para la Filantropía, A.C. Esta referencia ha sido utilizada no sólo por organizaciones (Mundo Ejecutivo, 2010), sino también por algunos investigadores (Pérez, 2008; Mercado, 2007).

Más allá de profundizar sobre el tema de la medición de la RSE en la agroindustria, este trabajo tiene como objetivo establecer la relación que existe entre RSE y competitividad de las empresas del sector agroindustrial.

Para Genier, Stamp y Pfitzer (2010) la RSE ha emergido como un área de acción para empresas grandes globales. Dado que el sector agroindustrial depende de recursos naturales, humanos y físicos, la innovación responsable es cada vez más vista en estas empresas; son dos tipos de iniciativas las que han surgido en la vanguardia de las empresas de este sector: 1) agendas de RSE (estándares y códigos, tales como Globalgap, Rainforest Alliance/SAN, SCS-001, Ethical Trading Initiative Fairtrade Standards, entre otros) y 2) innovaciones en la cadena de valor. No obstante, el compromiso de RSE no ha sido asumido por las empresas completamente: mientras algunas empresas actúan de "manera ética", obligadas por su cadena de valor, otras asumen el verdadero compromiso y ven en ella una oportunidad para crear valor (competitividad) a través de sus actividades, beneficiando así a todos los grupos de interés de la cadena de valor y a la comunidad en la cual operan (Genier et al., 2010).

\section{Metodología}

Anteriormente, se presentaron los fundamentos teóricos de la relación entre RSE y competitividad. A continuación, se presenta la manera en que se llevó a cabo la evaluación de la RSE de las empresas agrícolas y agroindustriales aguacateras del municipio de Uruapan, Michoacán, para posteriormente - en el apartado Resultadospoder generar una explicación sobre la relación con la competitividad del sector.

La evaluación de RSE se llevó a cabo utilizando, con unas modificaciones efectuadas por los autores, el modelo propuesto por el Centro Mexicano para la Filantropía A.C. (Cemefi); el cual se compone de cuatro planos (calidad de vida, ética empresarial, vinculación con la comunidad y medio ambiente), 27 dimensiones 
y 120 indicadores; fue utilizado porque corresponde con los estándares utilizados internacionalmente para evaluar la RSE, además de que ha sido empleado por algunos investigadores (Pérez, 2008; Mercado, 2007) y organizaciones (Mundo Ejecutivo, 2010) para medir la RSE en México.

El instrumento utilizado fue un cuestionario con escala de respuesta dicotómica, asignando el valor de 0 si las empresas no contaban con lo solicitado y valor de 1 en caso de contar con ello; su confiabilidad fue determinada con el Alfa de Cronbach que fue de $94 \%$. Se aplicó en doce empresas agrícolas y agroindustriales de aguacate de Uruapan, las cuales representan el $30 \%$ del total de empresas del sector de esta región. La información fue recabada de manera directa mediante entrevista personal durante el periodo de julio a diciembre de 2010.

\section{Índice de Responsabilidad Social Empresarial (IRSE)}

Con los datos obtenidos en el cuestionario de RSE y la opinión de los entrevistados respecto a de la importancia relativa de cada una de las cuatro dimensiones de la RSE, se elaboró un índice de responsabilidad social empresarial (IRSE); la contribución de los autores es la de haber utilizado un método de valoración multicriterio: proceso analítico jerárquico o Analytic Hierarchy Process (AHP, por su siglas en inglés) para determinar el peso relativo de cada aspecto, cuyo valor oscila entre cero y uno. Dicho método permite asignar valores a múltiples atributos a través de comparaciones pareadas (uno a uno) y permite considerar tanto factores objetivos como subjetivos de distinta índole, otorgando un peso o ponderación de acuerdo con la importancia relativa de cada atributo, variable o criterio incorporado al análisis; con lo que se garantiza un coeficiente de consistencia como prueba de bondad o ajuste del modelo de valoración construido. Para la realización de estas comparaciones se utilizan escalas de razón en términos de importancia, preferencia o probabilidad, sobre una escala numérica que va de uno hasta nueve (Saaty, 2008). En el cuadro 1 se muestran los valores obtenidos en el IRSE mediante el AHP, de acuerdo con las respuestas de las empresas entrevistadas. 


\section{Cuadro 1}

\section{Peso relativo de las cuatro dimensiones incorporadas al IRSE}

\begin{tabular}{l|c}
\multicolumn{1}{c|}{ Variable } & Valor \\
\hline Calidad de vida & 0.462 \\
\hline Ética empresarial & 0.178 \\
\hline Vinculación con la comunidad & 0.086 \\
\hline Medio ambiente & 0.24 \\
\hline IRSE & 1 \\
\hline
\end{tabular}

Posteriormente las cuatro variables fueron normalizadas para hacer las comparaciones entre ellas, otorgando así las siguientes categorías a los valores (ver cuadro 2).

\section{Cuadro 2}

Categorías otorgadas a las variables

\begin{tabular}{c|c}
\hline Rango & Categoría \\
\hline $0-0.200$ & Muy bajo \\
\hline $0.201-0.400$ & Bajo \\
\hline $0.401-0.600$ & Medio \\
\hline $0.601-0.800$ & Alto \\
\hline $0.801-1.00$ & Muy alto \\
\hline
\end{tabular}

\section{Resultados}

\section{Índice de Responsabilidad Social Empresarial}

De las doce empresas entrevistadas ninguna alcanzó el nivel más alto; el valor máximo fue de 0.750 , mientras que el mínimo fue de 0.2870 . Por lo que respecta a las medidas de tendencia central, la media fue de 0.5158 , que se interpreta como un nivel medio de RSE. En las medidas de dispersión, la varianza fue de 0.018 con una desviación estándar de 0.1344 .

En la figura 2 se presentan los resultados obtenidos en el IRSE por cada una de las empresas de acuerdo con el nivel obtenido. 
Figura 2

Resultados del IRSE en empresas agrícolas y agroindustriales de aguacate de Uruapan, Michoacán

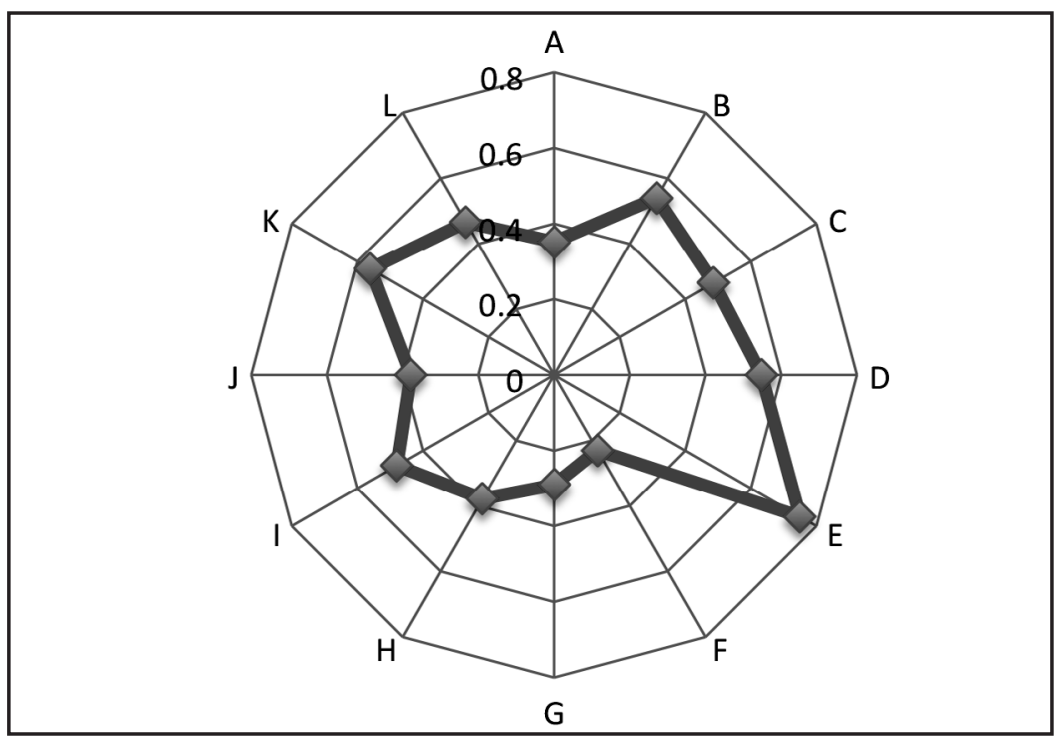

Fuente: Elaboración propia.

En la figura anterior se observa que ninguna empresa se ubicó en el nivel muy bajo; dos empresas (F y J) que corresponden al 17\% se ubicaron el nivel bajo; siete empresas (A, C, D, G, H, I) que representan el $58 \%$ se localizaron en el nivel medio; tres empresas (B, E, K) que conforman el $25 \%$ se ubicaron en el nivel alto; finalmente, ninguna empresa se ubicó en el nivel muy alto.

De lo anterior se concluye que diez empresas (83\%) se encuentran con niveles medianos y altos de RSE; esto es, las empresas están realizando acciones de RSE sin ser consideradas como tal y mucho menos está siendo considerado como una estrategia para las empresas. En el cuadro 3 se presentan los resultados obtenidos en las cuatro variables y las 27 dimensiones. 


\section{Cuadro 3 \\ Resultados promedio de las variables y dimensiones de RSE de las empresas agrícolas y agroindustriales aguacateras de Uruapan, Michoacán}

\begin{tabular}{|c|c|c|}
\hline \multirow{6}{*}{\multicolumn{2}{|c|}{$\begin{array}{c}\text { RSE } \\
(0.5158-\text { medio })\end{array}$}} & Empleabilidad (0.81) \\
\hline & & Gestión del capítal humano (0.79) \\
\hline & & Balance entre trabajo y familia $(0.51)$ \\
\hline & & Seguridad y salud laboral (0.86) \\
\hline & & Capacitación, educación y des. (0.44) \\
\hline & & Diversidad e igualdad de oport. (0.58) \\
\hline & \multirow[t]{8}{*}{ Ética empresarial 0.4785} & Derechos humanos $(0.75)$ \\
\hline & & Gobierno corporativo $(0.38)$ \\
\hline & & Compromiso con los stakeholder $(0.30)$ \\
\hline & & Compromiso ético $(0.26)$ \\
\hline & & Trato justo y globalidad $(0.63)$ \\
\hline & & Apertura y transparencia $(0.44)$ \\
\hline & & Alineación estratégica $(0.23)$ \\
\hline & & Combate a la corrupción $(0.43)$ \\
\hline & \multirow[t]{5}{*}{ Vinculación con la comunidad 0.2295} & Difusión de la RSE (0.25) \\
\hline & & $\begin{array}{c}\text { Ciudadanía y civismo empresarial } \\
(0.26)\end{array}$ \\
\hline & & Inversión social $(0.10)$ \\
\hline & & Balance social (0.02) \\
\hline & & Cadena de valor $(0.18)$ \\
\hline & \multirow[t]{8}{*}{ Medio ambiente 0.2415} & Operaciones ambientales $(0.23)$ \\
\hline & & Políticas ambientales $(0.17)$ \\
\hline & & Inversión (0.19) \\
\hline & & Capacitación y programas $(0.10)$ \\
\hline & & Información y comunicación $(0.14)$ \\
\hline & & Relaciones externas $(0.10)$ \\
\hline & & Transporte $(0.13)$ \\
\hline & & Manejo de impacto ambiental (0.05) \\
\hline
\end{tabular}


A continuación explicamos los resultados de cada una de las variables y dimensiones, así como las implicaciones que tienen estos resultados en la competitividad del sector.

\section{Calidad de vida}

El resultado máximo obtenido en esta variable fue de 0.8421 y el mínimo de 0.4211 . El resultado promedio fue de 0.6322 , que se traduce en un nivel alto. En lo que concierne a las medidas de dispersión, la desviación estándar fue de 0.1282 y la varianza de 0.0164 .

Al clasificar a las empresas de acuerdo con el nivel obtenido encontramos que ninguna obtuvo nivel muy bajo, ni bajo; cuatro (33\%) se ubicaron en un nivel medio, siete (58\%) en un nivel alto y una más (8\%) en el nivel muy alto. De lo anterior se concluye que $66 \%$ de las empresas evaluadas se clasifican como altas y muy altas en esta variable, lo que refleja un elevado nivel en el aspecto interno de la RSE de estas empresas.

Los dos aspectos mejor evaluados de esta variable fueron: 1) la salud y seguridad y 2) la empleabilidad; mientras que los dos más bajos fueron: 1) la capacitación, educación y desarrollo y 2) la diversidad e igualdad de oportunidades.

Por lo que respecta a la salud y seguridad laboral, se cumplió en $86 \%$, ya que las empresas afirmaron rechazar el uso de castigos corporales, evitar la coerción física o mental y abusos de jefes a subordinados, contar con un entorno laboral seguro, disponer de señalamientos para salidas de emergencia, promover hábitos de trabajo saludables y tener condiciones higiénicas para las necesidades básicas. Todos estos aspectos repercuten principalmente en la satisfacción del trabajador, generando con ello una mayor productividad en la empresa.

De los aspectos evaluados en la empleabilidad se encontró que $81 \%$ cumple con condiciones favorables en la composición de salarios y liquidaciones, respetan el derecho de los empleados a negociar en materia laboral y ofrecen beneficios básicos iguales a trabajadores de la empresa y a los contratados mediante outsurcing. Este resultado tiene efectos positivos en la rentabilidad de la empresa porque estas condiciones básicas permiten una estabilidad del empleado, con lo que se incurre en un menor gasto en la rotación del personal. 
Por lo que concierne a los valores bajos obtenidos, encontramos que con la capacitación, educación y desarrollo se cumplió únicamente en $44 \%$; destaca la falta de reconocimiento a las iniciativas de innovación del trabajador. Como consecuencia de ello se resta competitividad a la empresa porque no se estimula al trabajador a mejorar en su trabajo.

La diversidad e igualdad de oportunidades se cumplió únicamente en 58\%, pues las empresas no han incluido ni generado las condiciones necesarias para emplear a personas con capacidades diferentes o mayores de 50 años. La falta de estos elementos repercute en una menor competitividad de la organización en términos de su imagen y reputación como empresa social incluyente.

\section{Ética empresarial}

Por lo que respecta a esta variable, el valor máximo obtenido por las empresas entrevistadas fue de 0.8222 y el mínimo de 0.2444 . El resultado promedio obtenido fue de 0.4785 , el cual se traduce en un nivel medio. En lo que concierne a las medidas de dispersión, la varianza fue de 0.0383 y la desviación estándar de 0.1956.

Clasificando a las empresas por el nivel obtenido, ninguna empresa se ubicó en el nivel muy bajo; cuatro (33\%) se ubicaron en el nivel bajo, tres (25\%) se ubicaron en un nivel medio, cuatro (33\%) se ubicaron en el nivel alto y sólo una (8\%) calificó como muy alta. A diferencia de la calidad de vida, en donde los resultados se orientaron más en los niveles altos y muy altos, en la ética 58\% de las empresas se ubicaron en los niveles medio y alto.

Los aspectos mejor evaluados en esta variable fueron los derechos humanos y trato justo y globalidad, mientras que el compromiso ético y el compromiso con los grupos de interés fueron los aspectos evaluados como más bajos.

De acuerdo con los resultados obtenidos, $75 \%$ de las empresas entrevistadas establecen conocer y respetar los derechos humanos de sus colaboradores y su ámbito de influencia, sancionar la violación de ellos y evitar operaciones que vayan en contra de la dignidad de las personas. Esto genera una imagen positiva y una mejor reputación, lo que podría influir en el impacto en la demanda de consumidores que buscan tratos justos a los trabajadores. 
El trato justo y globalidad está relacionada directamente con la transparencia de las empresas. Las que fueron encuestadas cumplieron esta dimensión en un 63\%, con lo que se afirma que operan con prácticas de mercado honestas, transparentes, garantizando honradez en las transacciones y promoviendo prácticas de venta basadas en criterios éticos. Esto significa que al operar de manera ética y transparente puede existir un mayor número de inversionistas interesados en realizar inversiones en estas empresas, con lo que mejoran su competitividad.

De los elementos evaluados bajo esta variable, el compromiso ético se cumplió en $26 \%$, debido principalmente a que las empresas no tienen establecido un código de conducta o ética para diferentes tomas de decisiones; su falta tiene un efecto en la transparencia de la empresa.

El poco compromiso con los grupos de interés fue el segundo elemento más bajo evaluado de esta variable, cumpliendo sólo con el 30\%. Las razones fueron, en primer lugar, el desconocimiento de sus grupos de interés, seguido de la falta de un proceso para definir a dichos grupos, así como el involucramiento con ellos en conocer sus expectativas, buscar los mejores mecanismos de comunicación, etc. Estas debilidades presuponen un efecto negativo en las relaciones con el entorno, careciendo con ello de la comprensión de su ámbito competitivo.

\section{Vinculación con la comunidad}

El valor máximo obtenido en esta variable fue de 0.5833 y el mínimo de 0.0833 . El resultado promedio obtenido por las doce empresas fue de 0.2295 , el cual se traduce en un nivel bajo. En lo que concierne a las medidas de dispersión, la varianza fue de 0.0352 y la desviación estándar fue de 0.1876 .

Clasificando a las empresas por su nivel, a diferencia de la calidad de vida y ética empresarial, en la vinculación con la comunidad se encontraron resultados heterogéneos. Cuatro empresas (33\%) se ubicaron en el rango muy bajo, otras cuatro (33\%) calificaron con bajo nivel, otras cuatro más (33\%) calificaron con nivel medio y ninguna empresa calificó con nivel alto o muy alto. Por lo tanto, se concluye que $66 \%$ de las empresas analizadas se ubican en el rango bajo y muy bajo, lo que refleja una desarticulación de estas empresas con la sociedad, principalmente en su área geográfica de operación. 
A diferencia de las dos variables anteriores, en esta variable se obtuvieron valores bajos en los cinco aspectos evaluados. No obstante, los elementos que resultaron ser menos bajos fueron la ciudadanía y civismo empresarial y la difusión de la $R S E$, mientras que los elementos que resultaron más bajos fueron la inversión social y la cadena de valor.

En la ciudadanía y civismo empresarial se obtuvo un cumplimento del $26 \%$. Es importante mencionar que con este resultado las empresas afirmaron apoyar acciones de interés público para contribuir a reducir la desigualdad social y tomar una posición activa en la discusión y propuestas de políticas públicas, que cotidianamente se traducen en una participación activa hacia el exterior a través de las agrupaciones gremiales. En este sentido, tales actividades tendrían un efecto positivo en la imagen y reputación de las empresas dentro de toda la agroindustria o cadena de valor del aguacate, lo que influiría en la demanda por este producto.

El segundo elemento mejor evaluado fue la difusión de la RSE, que se cumplió en un $26 \%$. Es decir, las empresas contribuyen en el desarrollo social, ambiental y económico para la construcción de una sociedad sustentable en las comunidades en que operan; y participan en el fortalecimiento de las organizaciones locales que representan intereses legítimos de la comunidad. Estos elementos se relacionan directamente con la transparencia, lo cual puede influir en un aumento de inversionistas interesados en las empresas.

En lo que concierne a los aspectos más bajos, el que resultó menos favorable fue inversión social, pues sólo alcanzó un cumplimiento apenas del 10\% debido a la falta de un programa social y de vinculación a la comunidad, al poco interés por aportar recursos financieros, lo que se refleja en no destinar presupuesto para programas de interés público que contribuyan a la reducción de la desigualdad social o al desarrollo humano, y al poco interés por aportar recursos no financieros para el desarrollo de proyectos y actividades de la comunidad. Todos estos aspectos hacen que la sociedad o comunidad en la que operan tenga una imagen negativa de ellas, generando con ello una reducción de la demanda de consumo y una pérdida de competitividad.

La cadena de valor se cumplió en $18 \%$ debido a la ausencia de una estrategia de RSE en las empresas, así como de los integrantes de su cadena de valor, y a la falta de inclusión por parte de los proveedores de estas empresas a individuos, organizaciones sociales o grupos comunitarios usualmente excluidos (indígenas, 
personas con discapacidad, poblaciones rurales, etc.). Las debilidades presentadas en este aspecto generan una imagen y reputación negativa que puede influir en la demanda, así como una frágil relación con sus stakeholders que genera una incomprensión del entorno competitivo.

\section{Medio ambiente}

Por último, en esta variable el valor máximo obtenido por las doce empresas fue de 0.7805 y el mínimo de 0.0732 . El promedio fue de 0.2415 , que se traduce en un nivel bajo. En lo que concierne a las medidas de dispersión, la varianza fue de 0.0431 y la desviación estándar de 0.2076.

De manera similar a la vinculación con la comunidad, los resultados de las empresas analizadas reflejan poca atención a los aspectos ambientales tanto al interior como al exterior de la misma. Al clasificar a las empresas se encontró que cuatro (33\%) se ubicaron en el nivel muy bajo, cinco (42\%) se ubicaron en el nivel bajo, tres (25\%) se ubicaron en el nivel medio, una más se localizó en el nivel alto, mientras que ninguna empresa se ubicó dentro del rango de muy alto. De lo anterior se concluye que $75 \%$ de las empresas analizadas tienen un nivel bajo y muy bajo en los aspectos medioambientales.

A pesar de los resultados bajos obtenidos en esta variable, podemos destacar el desempeño en las operaciones ambientales y las inversiones ambientales como elementos bien evaluados. Por el contrario, los de evaluación más baja fueron el manejo de impacto ambiental y la capacitación y programas ambientales.

De acuerdo con los resultados obtenidos, las empresas estudiadas cumplieron con el $23 \%$ de los aspectos evaluados en las operaciones ambientales. Todas utilizan productos y tecnologías ambientales en distintas áreas y departamentos de la empresa; sólo algunas cuentan con un área dentro de la empresa exclusiva para los asuntos ambientales. Con estos elementos se genera imagen positiva de las empresas, tanto al interior como al exterior de las mismas, que puede influir en un aumento en la demanda de consumo.

La inversión ambiental se cumplió en 19\%. De aquí destaca el aprovechamiento alternativo (reutilización, reciclaje, donación, venta) de residuos, subproductos y desechos; la disposición adecuada de todo su inventario muerto de mobiliario y equipo; así como la realización de inversiones con el objetivo de reducir y sus- 
tituir recursos de entrada. Estas acciones tienen un efecto positivo en la imagen y reputación de la empresa de los diversos públicos, pero principalmente de los consumidores.

En lo que respecta al manejo de impacto ambiental, éste se cumplió únicamente en 5\%. La falta de realización de estudios de impacto medioambiental (independientemente de las obligaciones legales), la falta de estudios de impacto ambiental de la cadena productiva y del ciclo de vida de sus productos, el poco interés por la utilización de fuentes de energía renovable, la falta de procesos de gestión medioambiental estandarizados y formalizados, y como consecuencia la ausencia de algún reconocimiento por el interés en los temas ambientales, entre otros, dan como resultado una débil relación con los grupos de interés o stakeholders en términos de sostenibilidad, así como una imagen y reputación negativa de la empresa. Ambos aspectos afectan al entorno competitivo, así como a la demanda de consumidores con mayor conciencia ambiental, lo que se traduce en una pérdida de competitividad.

La capacitación y programas ambientales se cumplieron en un 10\%. Lo anterior se debió a la falta programas ambientales educativos de acceso público, a la falta de extensión de las políticas - utilizadas para la selección de proveedores - y criterios ambientales a la cadena de suministro, a la carencia de campañas de concienciación y educación medioambiental dirigidas a públicos más amplios, entre otros. Estos aspectos que no se están cumpliendo tienen un impacto en la imagen y reputación de la empresa, lo que influye en la demanda del consumidor quien está vigilando las acciones ambientales que realiza la empresa. Lo anterior genera, por lo tanto, una pérdida de competitividad derivado de estas carencias ambientales.

\section{Discusión de resultados}

Al no contar con una referencia teórica sobre la evaluación de la RSE agrícola en Michoacán, resulta complicado hacer una discusión de resultados basándonos en hallazgos previos de otras investigaciones. Sin embargo, en esta parte se hace una discusión en dos sentidos: la primera, sobre el modelo utilizado para medir la RSE y, la segunda, sobre los resultados y su relación con la competitividad.

Uno de los principales retos a los cuales se enfrenta actualmente la teoría de la RSE es su medición (Pasquero, 2005). Hasta hoy no existe un solo método para evaluar la RSE, ya que en el mundo existen más de 227 modelos de RSE que han sido 
elaborados por investigadores, universidades e instituciones, principalmente Gond (2008). En esta investigación se eligió el modelo de RSE del Centro Mexicano para la Filantropía (Cemefi), ya que correspondía no sólo a estándares internacionales y ha sido utilizado por otros estudios para medir la RSE en México (Pérez, 2008; Mercado, 2007; Mundo Ejecutivo, 2010).

A pesar de que el modelo Cemefi ha sido referencia para varias investigaciones, así como para el sector empresarial para otorgar el distintivo ESR, al utilizarlo se encontraron dos debilidades. La primera es que existe sesgo en algunas de las preguntas; la segunda, referente a su aplicación limitada solamente a empresas, omite la opinión de los stakeholders o grupos de relación. Sin embargo, al utilizar este modelo, adaptado con el uso del proceso analítico jerárquico, fue posible tener una referencia inicial o "fotografía" más precisa de la RSE del sector agrícola y agroindustrial del aguacate de Uruapan, Michoacán.

Con la metodología utilizada se encontró que las empresas estudiadas tuvieron un resultado de $51.58 \%$, lo que se traduce como un nivel medio de RSE; no obstante, $83 \%$ de las empresas alcanzaron niveles regulares y altos de RSE. Esto significa que estas empresas están cumpliendo con algunos de los estándares internacionales que las ubican como socialmente responsables; sin embargo, hay que entender que la RSE es una cuestión de grado en la que permanentemente existe el reto de seguir ascendiendo en el cumplimiento de las variables que la conforman y así mejorar su competitividad.

En los componentes del IRSE se encontró en las empresas lo siguiente: en calidad de vida un nivel alto (66\% en los niveles alto y muy alto), en ética empresarial un nivel regular (58\% en los niveles regular y alto), en la vinculación con la comunidad un nivel bajo (66.7\% entre los rangos de bajo y muy bajo) y en medio ambiente un nivel muy bajo (75\% entre los niveles bajo y muy bajo). Por lo tanto, las fortalezas se tuvieron en los aspectos internos, mientras que las debilidades se presentaron en los aspectos externos. Obviamente que el resultado también podría diferir al utilizar otro modelo de RSE y/u otro método de evaluación.

La segunda cuestión por discutir es su relación con la competitividad. Esta relación no es compartida por otros enfoques como el caso del enfoque ético-moralista, el cual establece que la RSE es un comportamiento moral que tiene el empresario sea con el mismo, sus trabajadores o sus partes interesadas, o del enfoque o contrac- 
tual, el cual establece que la RSE es obligación de las empresas y que la ciudadanía puede exigir un comportamiento más ético de las empresas (Gendron, 2000).

El enfoque que está de acuerdo con esta relación y que además la promueve es el estratégico. A pesar de tener grandes críticas por su orientación neoclásica, este enfoque ha tenido gran aceptación en el mundo empresarial y académico. Dentro de sus supuestos considera a la RSE como un elemento que permite generar una ventaja competitiva en la empresa. A partir de este enfoque teórico, en esta investigación fue posible realizar inferencias entre la RSE, sus variables y la competitividad.

De acuerdo con los resultados obtenidos, salud y seguridad laboral; la empleabilidad; el trato justo y globalidad; la difusión de la RSE; el respeto a los derechos humanos; la ciudadanía y civismo empresarial; así como la inversión ambiental son los elementos de RSE que generan efectos favorables en la competitividad de las empresas agrícolas y agroindustriales de aguacate de Uruapan, Michoacán.

En tanto, la falta de capacitación; educación y desarrollo; el poco compromiso con los grupos de interés; la ausencia del tema de la RSE en la cadena de valor; la falta de diversidad e igualdad de oportunidades laborales; la poca inversión social; la falta de inclusión de aspectos de RSE en la cadena de valor; así como un bajo manejo de impacto ambiental son los elementos de RSE que están restando competitividad las empresas mencionadas.

\section{Conclusiones}

La problemática ambiental y social que se ha generado con la producción de aguacate en la región de Uruapan, Michoacán, es una realidad que se ve reflejada en la pérdida de biodiversidad derivado del monocultivo de aguacate, en los efectos en la salud de los trabajadores y la población del uso excesivo de agroquímicos; así como en la degradación de los recursos naturales.

Si bien la RSE no es la solución total a lo señalado anteriormente, es una estrategia que puede contribuir a mitigar algunos de los factores que la provocan. En este sentido y considerando que la actividad aguacatera es una de las más importantes de esta región, en esta investigación se realizó una evaluación de RSE de doce empresas agrícolas y agroindustriales de aguacate; se encontraron resultados positivos en el Î́ndice de Responsabilidad Social Empresarial. Además, se identificaron 
las fortalezas y debilidades de las variables analizadas y se realizaron inferencias respecto de la competitividad del sector.

Una importante aportación de este trabajo es el desarrollo de un método de medición más preciso de la RSE, el cual mejora la precisión del modelo del Centro Mexicano de Filantropía, A.C., al que se incorporó un sistema de ponderación diferenciada con base en la importancia relativa que se logró establecer empleando un proceso analítico jerárquico. Se sugiere que este modelo y el IRSE puedan ser probados en otros sectores y contextos de evaluación.

A partir de los resultados obtenidos en esta investigación, se propone el diseño de una estrategia de RSE en el nivel empresa y nivel sector. En el nivel de la unidad económica se encontró que muchas de las empresas analizadas ya realizaban acciones de RSE, sin denominarse como tal, por lo que se considera que hace falta integrarlas. Para ello, es importante que se entienda el concepto, así como los distintos estándares internacionales que califican a una empresa como socialmente responsable. Los resultados de cada empresa ayudan a las mismas a identificar sus debilidades y sus fortalezas para poner mayor atención a las primeras y algunos aspectos que resultasen bajos en las fortalezas.

Para la estrategia sectorial de RSE se deberían considerar los resultados obtenidos en la evaluación, dando prioridad a los aspectos que resultaron más bajos, ya que son los que están generando una reducción en la competitividad de las empresas. Esta estrategia puede ser impulsada mediante alguna de las organizaciones que agremian los distintos eslabones de la cadena productiva del aguacate, tales como la Asociación de Productores y Empacadores de Aguacate, A.C., la Unión de Empacadores y Comercializadores de Aguacate de Michoacán, A.C., la Comisión Michoacana del Aguacate, entre otras.

La implementación de una estrategia de RSE no sólo beneficiaría a las empresas o al sector mediante el incremento de su competitividad, sino también generaría beneficios en el desarrollo de las comunidades con un enfoque sustentable. La RSE crea sinergia entre los grupos de interés de manera que los beneficios se expanden a estos grupos.

En particular, si las empresas agrícolas y agroindustriales de aguacate implementan la RSE estarán motivando a productores, proveedores de insumos y demás integrantes de la cadena de valor a operar bajo estos principios, con lo que generan 
un compromiso implícito, principalmente con el medio ambiente, principal problema de este sector, mediante la reducción de uso de agroquímicos y un mayor involucramiento con las comunidades en donde operan.

\section{Referencias}

Barsimantov, J. A. y A. Navia (2008). Land use and land tenure change in Mexico's avocado production region: Can community forestry reduce incentives to deforest for high value crops? Duodécima Conferencia Bienal de la Asociación Internacional para el Estudio de los Comunes. Disponible en http://journalogy.com/Paper/12152822.aspx

Becchetti, L. (2004). The impact of Social Responsibility on productivity and efficiency of US listed companies. XIII Tor Vergata Financial Conference. Roma: University of Rome "Tor Vergata".

Bowen, H.R. (1953). Social Responsibility of the Businessman. New York: Harper and Row.

Brine, M., R. Brown y G. Hackett (2007). Corporate Social Responsibility and Financial Performance in the Australian Context [online]. Economic Roundup otoño 2007: 47-58. Disponible en: http://search.informit.com.au/documentSummary; $\mathrm{dn}=254072917099846 ;$ res=IELBUS

Business Week (2007). Special Report, The 100 Top Brands. Disponible en: http:// www.businessweek.com/magazine/toc/07_32/B40450732bestbrands.htm

(2007). Special Report, The World's Most Innovative Companies. Disponible en: http://www.businessweek.com/innovate/di_special/ 20070503mostinnovat.htm

Business and Human Rights Resource Centre (2007). Business and Human Rights: Mapping International Standards of Responsibility and Accountability for Corporate Acts. Disponible en: www.business-humanrights.org.

Comisión Nacional de Áreas Naturales Protegidas, Conanp (2006). Programa de Conservación y Manejo Parque Nacional Barranca del Cupatitzio. Disponible en: http://www.conanp.gob.mx/anp/consulta/Barranca.pdf 
Donaldson, T. y L. E. Preston (1995). The Stakeholder Theory of the Corporation: Concepts, Evidence, and Implications. Academy of Management Review 20: 65-91.

Elkington, J. (1998). Cannibals with Folks:The tripple bottom line of 21st Century Business. New Society Publishers.

Fortune (2007). Fortune Most 'accountable' Companies. Disponible en: http:// money.cnn.com/magazines/fortune/global500/2007/accountability/full_list. html

Freeman, R. (1984). Strategic Management: A Stakeholder Approach. Cambridge: Cambridge University Press.

Friedman, M. (1970). The Social Responsibility of Business is to increase its Profits. New York Times Magazine (32-33): 122-126.

Garriga, E. y D. Melé (2004). Corporate Social Responsibility: Mapping the Territory. Journal of Business Ethics 53: 51-71.

Gendron, C. (2000). Le questionnement éthique et social de l'Enterprise dans la littérature managériale. Cahiers du CRISES. Québec (Working Papers Etudes theoriques. No. ET0004).

Genier, C., M. Stamp y M. Pfitzer (2010). Corporate Social Responsibility in the Agrifood Sector: Harnessing Innovation for Sustainable Development. FSG. Disponible en: http://www.fsg.org/tabid/191/ArticleId/296/Default. aspx?srpush=true

Gómez, F. (2006). Responsabilidad Social Corporativa y Performance Financiero: treinta y cinco años de investigación empírica en busca de un Consenso. XIV Congreso Nacional de Ética, Economía y Dirección. Disponible en: http:// www.eben-spain.org/docs/Papeles/XIV/GOMEZ_FRANCISCO.pdf

Gond, J. P. (2008). La Responsabilité Sociale de l'entreprise. Paris: Presses Universitaires de France. 
Great place to work (2011). ¿Qué es un excelente lugar de trabajo?. Disponible en: http://www.greatplacetowork.com.mx/nuestro-enfoque/ique-es-un-excelente-lugar-de-trabajo

Griffin, J. J. y J. F. Mahon (1997). The Corporate Social Performance and Corporate Financial Performance Debate. Business \& Society 36 (1): 5-31.

Hart, S. L. (1995). A Natural-Resource-Based View of the Firm. Academy of Management Review 20 (4): 986-1012.

y C. M. Christensen (2002). The Great Leap. Driving Innovation from the Base of the Pyramid. MIT Sloan Management Review 44 (1): 51-57.

ISO (2010). ISO 26000 project overview. Disponible en: http://www.iso.org/iso/ iso_26000_project_overview.pdf

Kissinger, G. (2012). Corporate social responsibility and supply agreements in the agricultural sector. Decreasing land and climate pressures. CCAFS Working Paper no. 14. CGIAR Research Program on Climate Change, Agriculture and Food Security (CCAFS). Copenhagen, Denmark. Disponible en: http:// www.ccafs.cgiar.org

Jensen, M. C. (2000). Value Maximization, Stakeholder Theory, and the Corporate Objective Function. En M. Beer y N. Nohria, eds. Breaking the Code of Change. Boston: Harvard Business School Press, Boston: 37-58.

Jones, Marc T. (1996). Missing the Forest for the Trees. A Critique of the Social Responsibility Concept and Discourse. Business \& Society 35 (1): 7-41.

Juscius, V. y V. Snieska (2008). Influence of Corporate Social Reponsibility on Competitive Abilities of Corporation. Engineering Economics 58 (3): 34-44.

Kay, J. (1993). Foundations of Corporate Success. Oxford: Oxford University Press.

Litz, R. A. (1996). A Resourced-Based-View of the Socially Responsible Firm: Stakeholder Interdependence, Ethical Awareness, and Issue Responsiveness as Strategic Assets. Journal of Business Ethics (15): 1355-1363. 
Mardensen, C. (2006). In defense of Corporate Responsibility. En A. Y. Kakabadse, ed. Corporate Social Responsiblitity. Recognition with application. New York: Palgrave.

Margolis, J. D. y J. P. Walsh (2003). Misery Loves Companies: Rethinking Social Initiatives by Business. Administrative Science Quarterly (48): 268-305.

McWilliams, A. (2006). Corporate social responsibility: strategic implications. Journal of Management Studies 43: 1-18.

Ouellet, M. L. y R. Audet (2004). Certifications et labels de l'agroalimentaire : environnement, équité, qualité et origine. Régulations et pratiques de la société civile, Recueil de textes CÉH/RT-19-2004 sous la direction de Corinne Gendron et Marie France Turcotte, UQAM, Canada.

Pasquero, J. (1995). L'environnement sociopolitique de l'Enterprise. La gestion des organisations: une approche systémique, conceptualle et stratégique. Montréal: Cheneliére/Mc Graw-Hill.

(2005). La responsabilité sociale de l'Enterprise comme objet des sciences de gestion. Un regard historique. En M. F. Turcotte y A. Salmon, eds. Responsabilité sociale et environnementale de l'Enterprise. Montréal : Presses de l’Université du Québec : 80-111.

Pedraza, H. (2004). Eficiencia administrativa y productividad en la industria eléctrica de la División Centro Occidente de México. Ciencia Nicolaita 37: 3959.

Porter, M. (2002). Ventaja Competitiva. México: CECSA.

y M. Kramer (2006). Strategy and Society: The link between Competitive Advantage and Corporate Social Responsibility. Harvard Business Review, december: 78-93.

Prahalad, C. K. (2002). Strategies for the Bottom of the Economic Pyramid: India as a Source of Innovation. Reflections: The SOL Journal 3 (4): 6-18. 
y A. Hammond (2002). Serving the World's Poor, Profitably. Harvard Business Review 80 (9): 48-58.

Propenko, J. (1991). La Gestión de la Productividad. México: Noriega-Limusa.

Saaty, T. L. (2008). Decision making with the analytic hierarchy process. Int. J. Services Sciences 1 (1): 83-98.

Schnietz, K. E. y M. J. Epstein (2005). Exploring the Financial Value of Reputation for Corporate Responsibility During a Crisis. Corporate Reputation Review 7 (4): 327-345.

Valor, C. (2005). Corporate Social Responsibility and Corporate Citizenship: Towards Corporate Accountability. Business and Society Review 110 (2): 191-213.

Villanova, M. (2009). Exploring the Nature of the Relationship Between CSR and Competitiveness. Journal of Business Ethics (87): 57-69. 
